\title{
Direct costs of chronic obstructive pulmonary disease among managed care patients
}

This article was published in the following Dove Press journal:

International Journal of Chronic Obstructive Pulmonary Disease

28 September 2010

Number of times this article has been viewed

\author{
Anand A Dalal' \\ Laura Christensen² \\ Fang Liu ${ }^{3}$ \\ Aylin A Riedel ${ }^{3}$ \\ 'US Health Outcomes, \\ GlaxoSmithKline, Research Triangle \\ Park, NC, USA; ${ }^{2}$ Health Economics \\ Outcomes Research, i3 Innovus, \\ Ann Arbor, MI, USA; ${ }^{3}$ Health \\ Economics Outcomes Research, \\ i3 Innovus, Eden Prairie, MN, USA
}

Correspondence:Anand A Dalal US Health Outcomes, GlaxoSmithKline, 5 Moore Dr, Mail Stop I7.I355B, Durham, NC 27709, USA

Tel +l 9194837286

Fax +19194830103

Email anand.a.dalal@gsk.com
Purpose: To estimate patient- and episode-level direct costs of chronic obstructive pulmonary disease (COPD) among commercially insured patients in the US.

Methods: In this retrospective claims-based analysis, commercial enrollees with evidence of COPD were grouped into five mutually exclusive cohorts based on the most intensive level of COPD-related care they received in 2006, ie, outpatient, urgent outpatient (outpatient care in addition to a claim for an oral corticosteroid or antibiotic within seven days), emergency department (ED), standard inpatient admission, and intensive care unit (ICU) cohorts. Patient-level COPDrelated annual health care costs, including patient- and payer-paid costs, were compared among the cohorts. Adjusted episode-level costs were calculated.

Results: Of the 37,089 COPD patients included in the study, 53\% were in the outpatient cohort, $37 \%$ were in the urgent outpatient cohort, $3 \%$ were in the ED cohort, and the standard admission and ICU cohorts together comprised 6\%. Mean (standard deviation, SD) annual COPD-related health care costs $(2008$ US\$) increased across the cohorts $(P<0.001)$, ranging from $\$ 2003$ $(\$ 3238)$ to $\$ 43,461(\$ 76,159)$ per patient. Medical costs comprised $96 \%$ of health care costs for the ICU cohort. Adjusted mean (SD) episode-level costs were \$305 (\$310) for an outpatient visit, \$274 (\$336) for an urgent outpatient visit, \$327 (\$65) for an ED visit, \$9745 (\$2968) for a standard admission, and $\$ 33,440$ for an ICU stay.

Conclusion: Direct costs of COPD-related care for commercially insured patients are driven by hospital stays with or without ICU care. Exacerbation prevention resulting in reduced need for inpatient care could lower costs.

Keywords: health care cost, health expenditure, lung diseases, managed care

\section{Introduction}

Chronic obstructive pulmonary disease (COPD) is a progressive disease defined by an airflow limitation that is not fully reversible. ${ }^{1}$ Patients with the disease exhibit pathologic features of emphysema and, to varying degrees, chronic bronchitis. COPD is further typified by periodic worsening of symptoms. Exacerbations, which can be life-threatening for certain patients, are characterized by an amplified inflammatory response and worsening of airflow. These changes in symptoms are sufficient to warrant a change in management and occur an average of approximately three times per year for patients with moderate to severe COPD. ${ }^{2}$ Exacerbations are associated with diminished quality of life for COPD patients, ${ }^{3,4}$ and their frequency and severity, as well as the presence of comorbid conditions and the number of medications needed to manage the disease, all contribute to COPD severity. ${ }^{1}$ 
COPD is highly prevalent in the $\mathrm{US}^{5,6}$ and worldwide. ${ }^{7,8}$ The US prevalence ${ }^{6}$ was estimated at 10 million adults in 2000 , and in 2007, 5.8\% of individuals aged 45-64 years had physician-diagnosed COPD. ${ }^{5}$ COPD is a leading cause of death in the US, and related mortality has been reported to be greater for men than for women, and mortality rates are highest for white patients, followed by black, Hispanic, and Asian individuals. ${ }^{5}$

Management of stable COPD is intended to prevent and control symptoms and to reduce the frequency and severity of exacerbations. ${ }^{1,9,10}$ Maintenance medications for COPD include long- and short-acting bronchodilators, and inhaled corticosteroids for patients with repeated exacerbations. Fixed-dose combination therapies include short-acting beta agonist (SABA)/anticholinergic bronchodilator combinations, and long-acting beta agonist (LABA)/corticosteroid combinations. ${ }^{1}$ Patients with very severe COPD might receive oxygen therapy. Medications are generally added as the disease progresses.

Exacerbations necessitate intensified management. Rescue medication can be started at home, but if the exacerbation is not well managed, hospitalization may be required. ${ }^{1,9}$ Patients with severe exacerbations might be managed in the emergency department (ED) or hospital, but life-threatening exacerbations may require immediate intensive care unit (ICU) admission. ${ }^{1}$ Treatment for exacerbations includes increasing the dose or frequency of SABA administration, adding an anticholinergic bronchodilator, administering oral or intravenous corticosteroids, or oxygen therapy. Because exacerbations can be caused by bacterial infection, antibiotics might also be given. These events (ED visit, hospitalization) and changes in treatment (SABA, oral/intravenous corticosteroids, antibiotics) are strongly associated with COPD exacerbation and have been used to identify exacerbations in clinical research. ${ }^{11-14}$

The high prevalence and morbidity of COPD contributed to a projected $\$ 29.5$ billion in related direct costs in 2010 in the $\mathrm{US}^{5}$ and the annual health care cost was estimated at $\$ 4119$ per COPD patient in $2000 .{ }^{15}$ Hospitalization is a major cost driver in COPD management in various health care systems, ${ }^{5,14,16-18}$ with hospital care projected to account for $45 \%$ of direct COPD costs in the US in $2010 .^{5}$ Prescription drugs comprise approximately $20 \%$ of COPD costs. ${ }^{5}$ Disease ${ }^{16}$ and exacerbation ${ }^{14,17}$ severity have both been identified as COPD cost drivers. However, published data appear to be lacking regarding costs for US commercially insured patients and US costs according to COPD severity.
Our objective was to estimate direct costs of COPD-related care for commercially insured patients in the US, including both health plan- and patient-paid amounts. Specifically, we wanted to determine the overall costs generated by patients who received care of different intensities, and the costs of different types of COPD-related health care visits ranging from outpatient visits to ICU stays. We also extrapolated the mean annual direct health care cost of COPD for the US managed care population.

\section{Material and methods Data sources}

Data for this retrospective analysis were obtained from a managed care claims database which included geographically diverse commercial health plan members in the US. The database included data for approximately 14 million commercial enrollees during the study period. Enrollment information and medical and pharmacy claims from calendar year 2006 were used in the analysis. In order to extrapolate cost information from the database population to all commercially insured patients in the US, data from the 2006 Medical Expenditure Panel Survey (MEPS), the most recent available, were also used. ${ }^{19}$ MEPS is a set of nationally representative US surveys that collect information on health service use, including demographic characteristics and health insurance coverage.

\section{Patient selection and cohort formation}

Commercially enrolled patients with evidence of COPD from January 01, 2006 through December 31, 2006 were included in the study population. COPD patients were identified in one of the following ways: an ED or inpatient facility claim with COPD (ICD-9-CM code 491, 491.0, 491.1, 491.2x, 491.8, $491.9,492,492.0,492.8,496)$ in the primary diagnosis position (the diagnosis code must have been on a hospital claim for an inpatient stay); an outpatient (routine or urgent care) claim with a primary diagnosis of COPD and a second medical claim with a COPD diagnosis in any position on a separate service date; or a physician claim with a primary or secondary diagnosis of COPD and a filled prescription for an anticholinergic, LABA, or inhaled corticosteroid/LABA combination. Eligible patients were aged 40 years or older as of 2006 and were continuously enrolled in the health plan with medical and pharmacy benefits during the year-long study period. Patients were also required to have race/ ethnicity information available. All data were deidentified and accessed with protocols compliant with the Health Insurance 
Portability and Accountability Act. ${ }^{20}$ Privacy board approval was obtained for use of race/ethnicity data.

Patient demographic data, including age, gender, and geographic region were collected from enrollment information. Information on comorbidities, including calculation of the Quan-Charlson comorbidity score ${ }^{21}$ and a flag for asthma diagnosis (ICD-9-CM 493.xx), and on fills of respiratory medications (based on pharmacy and medical claims) was collected to characterize further the patients in the study sample.

Patients were grouped into five mutually exclusive cohorts based on the most intensive type of COPD-related care they had received during the study year. Patients in the "outpatient" cohort had at least one medical claim for office or outpatient care with COPD indicated in the primary or secondary position, but no evidence of an urgent visit (defined below), ED visit, or inpatient stay for COPD. Outpatient care included physician office visits, outpatient hospital services, laboratory visits, and urgent care center visits. Patients in the "urgent outpatient" cohort had at least one medical claim for outpatient care for COPD (primary or secondary position) followed by a pharmacy claim for an oral corticosteroid or antibiotic within seven days after the visit, but no evidence of more serious care. Patients in the "ED" cohort had at least one medical claim for an ED visit for COPD (primary position) but no evidence of an inpatient stay for COPD. The "standard admission" cohort comprised patients with at least one inpatient stay for COPD (COPD diagnosis in the primary position on a hospital claim), but no evidence of ICU treatment during any inpatient stay. Patients in the "ICU" cohort had evidence of ICU care during a COPD inpatient stay during the study period. ICU care was defined as a medical claim during an inpatient stay with revenue code of $020 x, 021 x, 0223$ or 0234 or a current procedural terminology procedure code for critical care (99291-99292). These cohort definitions reflect COPD health care encounters associated with regular follow-up and exacerbations of increasing severity. ${ }^{1,11,13}$

For an additional patient-level cost analysis, study patients were grouped into cohorts based on race/ethnicity as indicated in enrollment information (African American, Caucasian, Hispanic, other/unknown).

\section{Cost calculations}

Cost calculations were based on total paid amounts in 2006, including patient- and health plan-paid amounts, as well as estimated Medicare or other payer contributions based on coordination of benefits information. To enable comparisons with current literature, patient- and episode-level costs, as well as costs used in the extrapolation calculation, were adjusted to 2008 US dollars using the annual medical care component of the Consumer Price Index. ${ }^{22}$

Patient- and episode-level costs associated with COPDrelated (COPD diagnosis code on a claim associated with the visit) outpatient visits, urgent outpatient visits, ED visits, standard admissions (no ICU), and ICU stays were identified. The criteria used to identify these types of visits were the same as those used to define the patient cohorts. Costs associated with COPD-related care of any intensity that was received by each patient, including the cohort-determining episode type and less serious visits, were included in the analyses. The number of care episodes was counted. For ambulatory services, one visit per day per provider was allowed.

COPD-related pharmacy costs were summed from pharmacy claims for any of the following medications: SABAs, LABAs, anticholinergics, methylxanthines, oral/intravenous corticosteroids, inhaled corticosteroids, inhaled corticosteroid/LABA combination, other respiratory medication, or antibiotics.

In the patient-level analysis, mean annual total health care costs, as well as costs for each type of COPD-related visit, other COPD-related medical services (from claims with COPD diagnosis in the primary position that did not fall into the specified categories), and medications were calculated for each of the patient cohorts. Patient-level costs were also calculated for race/ethnicity groups.

Costs per COPD-related care episode in 2006 were tabulated based on all episodes of a given type for each cohort during the year, and adjusted episode-level costs were estimated using multivariate models. Generalized linear models with gamma distribution and log link were used to account for the skewed distribution of costs. ${ }^{23}$ The models of ED and inpatient episode-level costs were adjusted for gender, age category, geographic region, race/ethnicity, Quan-Charlson comorbidity score, and asthma diagnosis. The models of episode-level outpatient costs were adjusted for the variables listed above, as well as COPD diagnosis code specific to the visit, provider type, and season of episode.

A weighted average of total direct annual COPD-related costs (medical and pharmacy) for the US managed care population was extrapolated, based on cost estimates from the sample represented in the study database and demographic data from 2006 MEPS. 


\section{Statistical analysis}

Patient-level cost distributions were compared among cohorts using the $t$-test for continuous variables and the Chi-square test for proportions and categoric variables. A $P<0.05$ was used to define statistical significance. The multivariate models were fitted by STATA 10.0 (StataCorp, College Station, TX) and all other analyses were conducted using SAS 9.1 (SAS Institute, Cary, NC).

\section{Results}

\section{Sample characteristics}

Descriptive characteristics of the patient cohorts are shown in Table 1. Based on the distribution of patients in the cohorts, approximately $90 \%$ of eligible COPD patients did not have an ED or inpatient visit during the study year. The source database population comprises heavily Caucasian individuals and patients in the South region, and the racial and geographic distribution of the database is reflected among COPD patients included in the study, as shown in Table 1. Most patients in each cohort were aged 50-64 years. Age differed significantly among cohorts, with mean age youngest in the urgent outpatient cohort and oldest in the standard admission cohort.

Quan-Charlson comorbidity score differed significantly among the care-intensity cohorts, with the lowest mean scores in the outpatient cohorts and the highest mean score in the ICU cohort (Table 1). Comorbidity scores also differed significantly by race/ethnicity $(P<0.001)$, Hispanic patients had the lowest mean (standard deviation, SD) score at 2.2 (1.8), Caucasian patients had a mean score of 2.3 (1.8), and African American patients had the highest mean score at $2.5(2.0)$.

As expected, the percentage of each cohort that used each type of medication during the study year generally increased across cohorts (Table 1). Almost half of the patients in the study sample received a SABA at some point in 2006, with the lowest percentages in the outpatient cohorts. Oral/intravenous corticosteroid receipt ranged from $30 \%$ of patients in the outpatient cohort to $76 \%$ of patients in the ICU cohort. Oxygen therapy use increased across the cohorts, from $14 \%$ of the outpatient cohort to $57 \%$ of the ICU cohort. Approximately $35 \%$ of patients used more than one medication class during the study year and $17 \%$ of all patients used no maintenance treatment (inhaled corticosteroid, LABA, inhaled corticosteroid/ LABA combination, anticholinergic, methylxanthine), but did receive rescue medication (ie, SABA, oral/intravenous corticosteroid, antibiotic).

\section{Types of visits}

Without regard to cohort, the COPD patients included in the study had a mean (SD) of 2.7 (2.9) outpatient visits, 0.7 (1.2) urgent outpatient visits, 0.08 (0.6) ED visits, and 0.05 (0.3) standard admissions during 2006.

The patient cohorts were defined based on the most intensive level of care received, but patients also had visits of less serious types. Within each cohort, outpatient visits were the most frequently observed type, with the mean count for the study year ranging from 2.5 (3.2) for the urgent outpatient cohort to 4.6 (4.6) for the ICU cohort $(P<0.001$ across cohorts). The mean count of standard admissions ranged from 0.2 (0.6) for the ICU cohort to $1.1(0.5)$ for the standard admission cohort $(P<0.001)$. Among patients who had an inpatient stay (ie, patients in the standard admission or ICU cohorts), the mean duration of a standard (non-ICU) inpatient stay was 10.2 (21.1) days. The ICU cohort averaged $1.1(0.4)$ ICU visits in 2006, and the mean duration of an ICU stay was 1.9 (2.9) days.

\section{Patient-level costs of COPD}

As shown in Figure 1, mean (SD) total annual patient-level COPD-related costs increased across the cohorts, ranging from $\$ 2003$ (\$3238) to $\$ 43,461(\$ 76,159)$. Medical costs, which include costs associated with health care visits, comprised the largest portion of COPD-related costs across all cohorts. Mean total medical costs increased substantially across cohorts (from $\$ 1004$ per year for the outpatient cohort to $\$ 41,727$ per year for the ICU cohort; $P<0.001$ among cohorts) but mean pharmacy costs per year changed relatively little (from $\$ 999$ for the outpatient cohort to $\$ 1734$ for the ICU cohort; $P<0.001$ among cohorts). Thus, medical costs accounted for a progressively greater percentage of total COPD-related health care costs; up to $90 \%$ for the standard admission cohort and $96 \%$ for the ICU cohort. All together, study patients generated $\$ 101,284,860$ in COPD-related medical costs in 2006 and $34 \%$ of that amount was attributable to the ICU cohort. Thus, although patients in the ICU cohort comprised $2.2 \%$ of the COPD population eligible for the study, they accounted for the greatest percentage of COPD-related medical costs.

Mean annual patient-level costs of each type of COPDrelated visit are shown in Figure 2. Costs associated with each type of visit differed significantly among the applicable cohorts ( $P<0.001$ for all among-cohort comparisons). As expected, mean expenditures associated with hospitalization and ICU care were substantially greater than costs for other types of visits. As shown in Figure 3, mean total 
Table I Characteristics of COPD patients by utilization cohort ${ }^{\mathrm{a}}$

\begin{tabular}{|c|c|c|c|c|c|c|c|}
\hline & $\begin{array}{l}\text { Total } \\
(n=37,089)\end{array}$ & $\begin{array}{l}\text { Outpatient } \\
\text { cohort }^{\mathrm{b}} \\
(\mathrm{n}=\mid 9,64 I)\end{array}$ & $\begin{array}{l}\text { Urgent } \\
\text { outpatient } \\
\text { cohort }^{c} \\
(n=13,833)\end{array}$ & $\begin{array}{l}\text { ED cohort }^{d} \\
(n=I 23 I)\end{array}$ & $\begin{array}{l}\text { Standard } \\
\text { admission } \\
\text { cohort }^{\mathrm{e}} \\
(\mathrm{n}=1547)\end{array}$ & $\begin{array}{l}\text { ICU cohort }^{f} \\
(n=837)\end{array}$ & $P$ value \\
\hline Male, n (\%) & $|8,12|(48.9)$ & $10,149(51.7)$ & $6300(45.5)$ & $586(47.6)$ & $666(43.1)$ & $420(50.2)$ & $<0.001$ \\
\hline \multicolumn{8}{|l|}{ Ethnicity, n (\%) } \\
\hline Caucasian & 31,247 (84.3) & 16,469 (83.9) & II,742 (84.9) & $1023(83.1)$ & $1312(84.8)$ & 701 (83.8) & 0.081 \\
\hline African American & $1958(5.3)$ & $1054(5.4)$ & $650(4.7)$ & $86(7.0)$ & $109(7.1)$ & $59(7.1)$ & $<0.001$ \\
\hline Hispanic & $1249(3.4)$ & $679(3.5)$ & $476(3.4)$ & $45(3.7)$ & $31(2.0)$ & I8 (2.2) & 0.008 \\
\hline Other/unknown & $2635(7.1)$ & $1439(7.3)$ & $965(7.0)$ & $77(6.3)$ & $95(6.1)$ & $59(7.1)$ & 0.254 \\
\hline \multicolumn{8}{|l|}{ Region, n (\%) } \\
\hline Northeast & 4155 (II.2) & $2419(12.3)$ & I355 (9.8) & $107(8.7)$ & $197(12.7)$ & $77(9.2)$ & $<0.001$ \\
\hline Midwest & $9988(26.9)$ & $525 I$ (26.7) & $3664(26.5)$ & $391(31.8)$ & $46 I(29.8)$ & $221(26.4)$ & $<0.001$ \\
\hline South & I6,807 (45.3) & $8537(43.5)$ & $6630(47.9)$ & $566(46.0)$ & $684(44.2)$ & $390(46.6)$ & $<0.001$ \\
\hline West & $6139(16.6)$ & $3434(17.5)$ & $2184(15.8)$ & $167(13.6)$ & $205(13.3)$ & $149(17.8)$ & $<0.001$ \\
\hline \multicolumn{8}{|l|}{ Age, n (\%) } \\
\hline $40-49$ & $4449(12.0)$ & $2262(11.5)$ & $1900(13.7)$ & $132(10.7)$ & $105(6.8)$ & $50(6.0)$ & $<0.001$ \\
\hline $50-64$ & $18,545(50.0)$ & $9645(49.1)$ & $7239(52.3)$ & $621(50.5)$ & $656(42.4)$ & $384(45.9)$ & $<0.001$ \\
\hline $65-74$ & $7225(19.5)$ & $3925(20.0)$ & $2572(18.6)$ & $233(18.9)$ & 304 (19.7) & 191 (22.8) & 0.003 \\
\hline$\geq 75$ & $6870(18.5)$ & 3809 (19.4) & $2122(15.3)$ & $245(19.9)$ & $482(31.2)$ & $212(25.3)$ & $<0.001$ \\
\hline $\begin{array}{l}\text { Asthma } \\
\text { diagnosis, n (\%) }\end{array}$ & $12,150(32.8)$ & $5620(28.6)$ & 5059 (36.6) & $545(44.3)$ & $602(38.9)$ & $324(38.7)$ & $<0.001$ \\
\hline $\begin{array}{l}\text { Quan-Charlson } \\
\text { score, mean (SD) }\end{array}$ & $2.3(1.8)$ & $2.3(1.7)$ & $2.3(1.8)$ & $2.7(2.1)$ & $3.1(2.2)$ & $3.8(2.4)$ & $<0.001$ \\
\hline \multicolumn{8}{|l|}{ Medication ${ }^{8}, \mathrm{n}(\%)$} \\
\hline SABA & I7,486 (47.2) & 7490 (38.1) & 7668 (55.4) & $811(65.9)$ & $996(64.4)$ & $521(62.3)$ & $<0.001$ \\
\hline LABA & $2039(5.5)$ & $979(5.0)$ & $816(5.9)$ & $72(5.9)$ & $110(7.1)$ & $62(7.4)$ & $<0.001$ \\
\hline Anticholinergics & $19,650(53.0)$ & $9435(48.0)$ & $7810(56.5)$ & $755(6 \mid .3)$ & $1047(67.7)$ & $603(72.0)$ & $<0.001$ \\
\hline Methylxanthines & $2000(5.4)$ & $782(4.0)$ & $843(6.1)$ & $120(9.8)$ & $155(10.0)$ & $100(12.0)$ & $<0.001$ \\
\hline $\begin{array}{l}\text { Oral//V } \\
\text { corticosteroids }\end{array}$ & $|8,25|(49.2)$ & $5889(30.0)$ & $9676(69.9)$ & $930(75.5)$ & II $23(72.6)$ & $633(75.6)$ & $<0.001$ \\
\hline ICS & $460 \mathrm{I}(12.4)$ & $2033(10.4)$ & 1994 (14.4) & $193(15.7)$ & $24 I(15.6)$ & $140(16.7)$ & $<0.001$ \\
\hline $\begin{array}{l}\text { ICS + LABA } \\
\text { combination }\end{array}$ & $15,736(42.4)$ & 7838 (39.9) & $6359(46.0)$ & $5 \mid 3(4 \mid .7)$ & $64 \mid(4 I .4)$ & $385(46.0)$ & $<0.001$ \\
\hline $\begin{array}{l}\text { Other respiratory } \\
\text { medications }\end{array}$ & $6042(16.3)$ & $267 \mid(13.6)$ & $2696(19.5)$ & $263(21.4)$ & $256(16.5)$ & $156(18.6)$ & $<0.001$ \\
\hline Oxygen therapy & $6986(18.8)$ & $2834(14.4)$ & $2522(18.2)$ & $408(33.1)$ & $742(48.0)$ & $480(57.3)$ & $<0.001$ \\
\hline Antibiotics & $27,230(73.4)$ & II,I 62 (56.8) & I3,043 (94.3) & $1045(84.9)$ & $1287(83.2)$ & $693(82.8)$ & $<0.001$ \\
\hline $\begin{array}{l}\text { Nonrespiratory } \\
\text { medications }\end{array}$ & $34,859(94.0)$ & |8,039 (9|.8) & I3,473 (97.4) & II 42 (92.8) & 1419 (91.7) & 786 (93.9) & $<0.001$ \\
\hline
\end{tabular}

Notes: aPatients were grouped into five mutually exclusive cohorts based on the most intensive type of COPD-related care they received; ${ }^{\mathrm{b}} \mathrm{Cohort}$ comprising patients with at least one medical claim for outpatient care and no more intensive care; ' ${ }^{\mathrm{C}}$ Cohort comprising patients with at least one medical claim for outpatient care for COPD followed by a pharmacy claim for an oral corticosteroid or antibiotic within seven days and no more intensive care; ${ }^{\circ}$ Cohort comprising patients with at least one medical claim for an

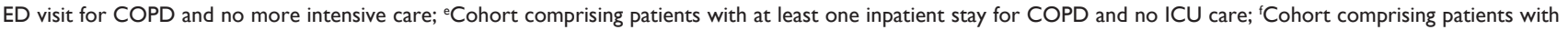
evidence of ICU care during an inpatient stay; ${ }^{\mathrm{B} A t}$ least one fill during the observation period.

Abbreviations: ED, emergency department; ICS, inhaled corticosteroid; ICU, intensive care unit; IV, intravenous; LABA, long-acting beta agonist; SABA, short-acting beta agonist; SD, standard deviation, COPD, chronic obstructive pulmonary disease.

COPD-related health care costs were approximately $21 \%$ to $25 \%$ lower for Hispanic patients than for patients of other races (among-group $P=0.135$ ).

\section{Episode-level costs of COPD}

The adjusted mean costs of a health care episode of each type are shown in Figure 4. Episode costs increased for more serious types of visits, with the most notable difference between outpatient/ED visits and inpatient/ICU visits. The adjusted mean cost of a COPD-related standard admission among all patients who had one was $\$ 9745$, and an ICU stay increased the cost to $\$ 33,440$ per episode.

\section{Population-level cost of COPD}

Based on MEPS data and data from this study, the 2006 prevalence of COPD among commercially insured patients 


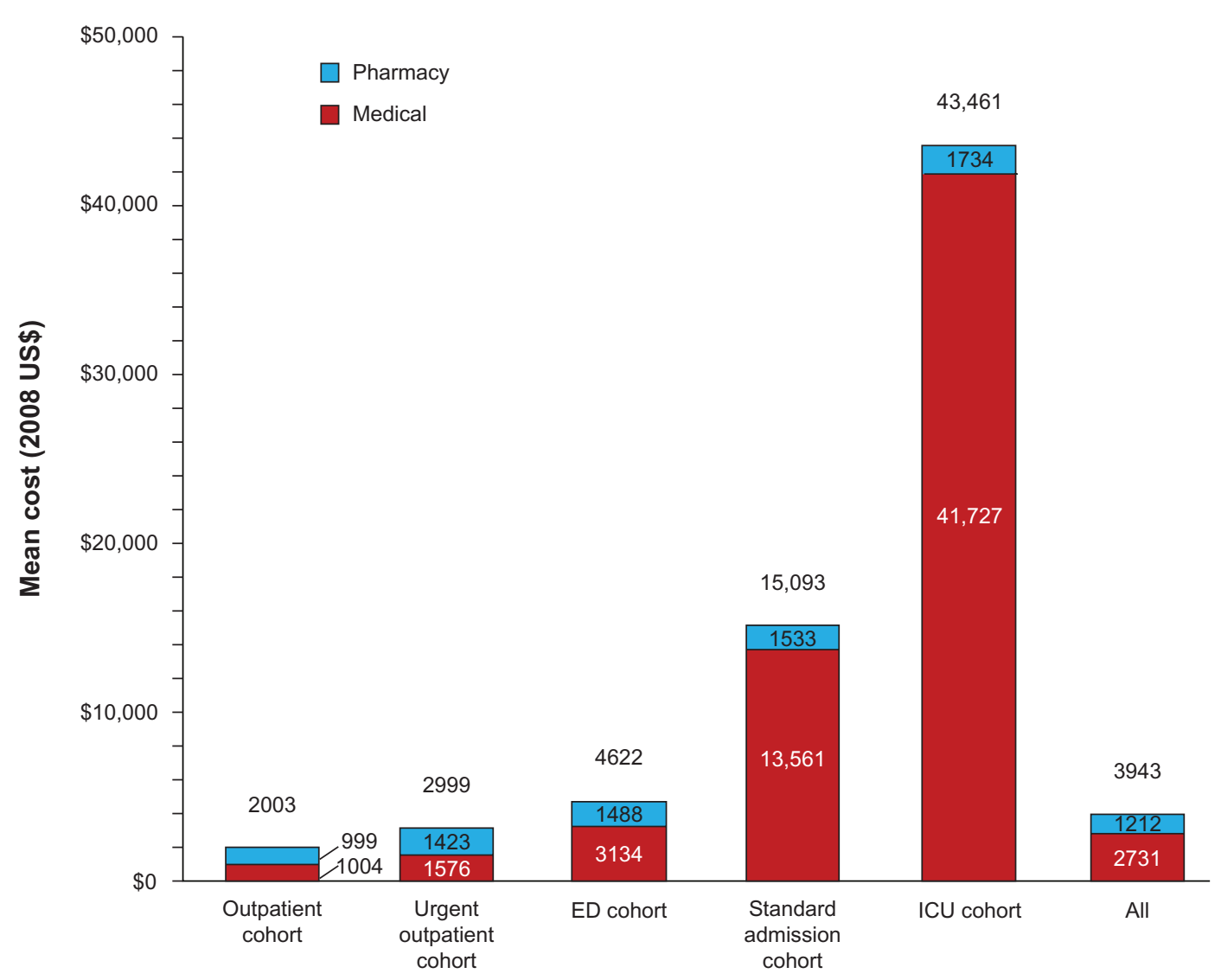

Figure I Mean annual COPD-related medical, pharmacy, and total costs by care intensity cohort. $P<0.00 \mathrm{I}$ among cohorts for medical costs, $P<0.00 \mathrm{I}$ among cohorts for pharmacy costs, and $P<0.001$ among cohorts for total COPD-related costs.

Abbreviations: ED, emergency department; ICU, intensive care unit; COPD, chronic obstructive pulmonary disease.

in the US was estimated at 2.9 million. This population was predicted to generate $\$ 15.7$ billion (in 2008 US\$) in annual direct health care costs, comprising approximately $\$ 12.4$ billion in medical costs and $\$ 3.3$ billion in COPD-related pharmacy costs.

\section{Discussion}

Our study provides estimated episode-level COPD-related encounter costs, including costs of an outpatient visit, ED visit, standard hospitalization and hospital admission resulting in an ICU stay, as well as patient-level direct annual COPD-related health care costs. The results show that costs increase according to the intensity of care received and expand on previous studies with the inclusion of commercial managed care patients, outpatient encounters, and costs stratified according to resource use suggestive of a range of COPD severities.

Stanford et $\mathrm{a}^{24}$ and Dalal et $\mathrm{al}^{25}$ provided hospitalperspective episode-level costs of encounters for a national sample of COPD patients for 2001 and 2005 through 2008. These previous studies reported that ICU visits, and intubation specifically, accounted for a small proportion of
COPD-related encounters, but a high proportion of costs. Costs associated with an ICU stay were approximately triple the costs of a standard admission for their national samples of patients with various health care payers, as well as for the commercial managed care patients in our study. Consistent with their hospital-perspective observations, ${ }^{24,25}$ we found that the ICU cohort generated a disproportionate share of health care costs from the perspective of patients and health plan payers. The cost of an ICU episode was much higher than costs for other types of visits, and mean annual health care costs were also substantially greater for patients who had an ICU encounter. These studies suggest that minimizing the need for hospitalization, and the ICU in particular, could lower COPD-related health care costs.

As guidelines advocate ${ }^{1}$ and observational studies bear out, ${ }^{2,14}$ COPD exacerbations can necessitate intensified medical resource use. Our findings are consistent with reports indicating that escalated use of medical resources drives COPD-associated health care costs. ${ }^{5,14-18}$ Our results further suggest that care indicative of more severe exacerbations (ie, hospitalization and ICU care) is associated with higher episode- and patient-level costs for patients 


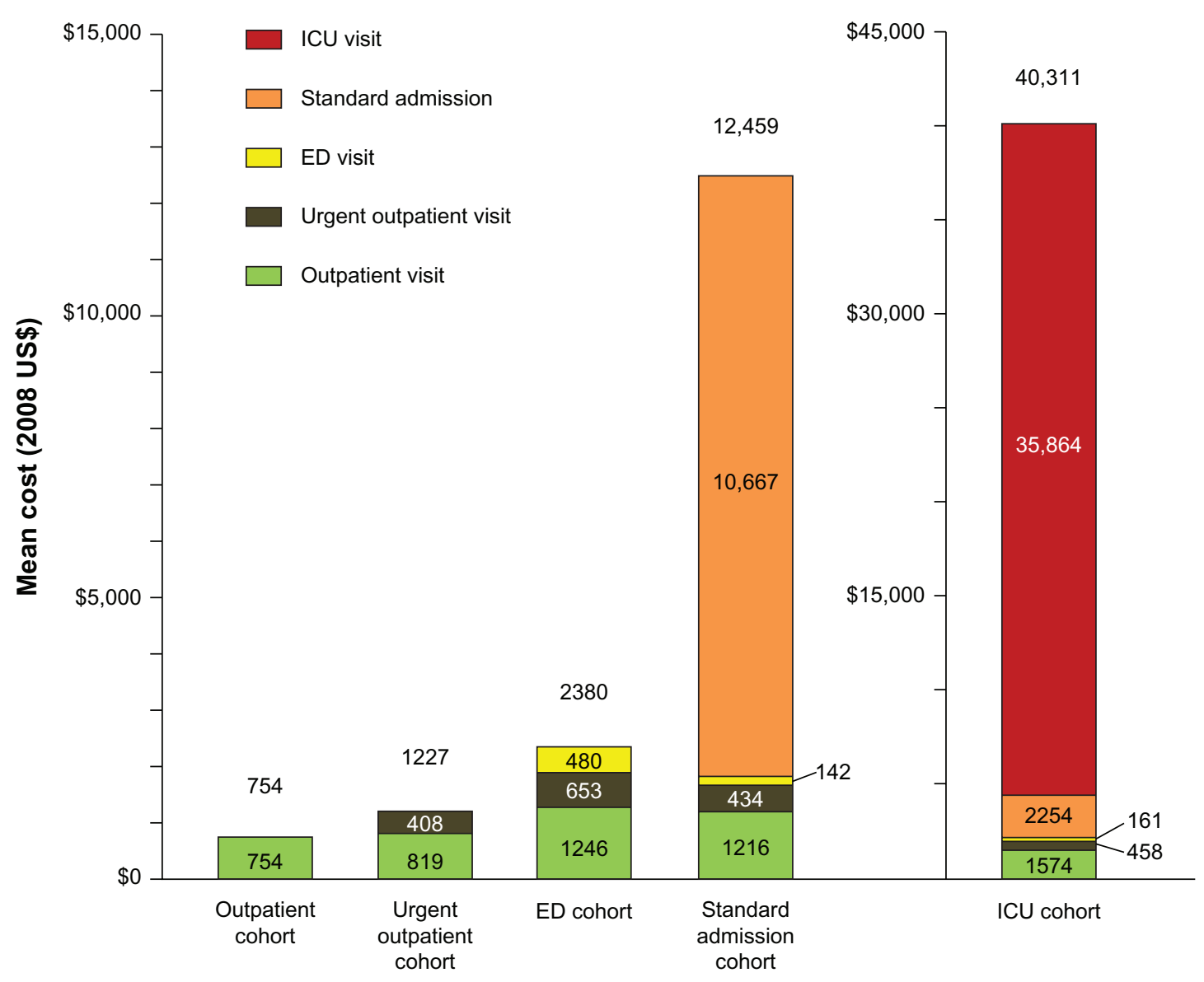

Figure 2 Mean annual patient-level cost of COPD-related visits by care intensity cohort. Among-cohort $P<0.001$ for costs associated with each type of visit. Abbreviations: ED, emergency department; ICU, intensive care unit; COPD, chronic obstructive pulmonary disease.

in the US. A Canadian study of COPD-related health care encounters also determined that severe exacerbations requiring hospitalization were associated with substantially higher episode-level costs than moderate-severity exacerbations, defined according to treatment in an outpatient or ED setting. ${ }^{14}$ Disease management initiatives targeted to preventing exacerbations may reduce health care costs by minimizing COPD-related need for inpatient resources. ${ }^{26}$ In addition, previous research suggests that reducing exacerbation frequency may improve patient quality of life. ${ }^{3}$

The results presented here must be interpreted in the context of certain limitations. As for all claims-based studies, it should be noted that claims are collected for payment, not research, and the analysis is constrained by the codes and other information contained in claims. If, for example, a critical care code was present on a claim for care that did not actually occur in the ICU, then ICU episodes might be overestimated based on our criteria. However, we believe that such cases are rare, and that the codes used to identify episode types provide a reasonable estimate of the range of care received by COPD patients. Claims data provide limited detail regarding in-hospital medication use. Because oxygen is often administered in the inpatient setting, this could account for the unexpectedly low proportion of patients in the ICU cohort that had evidence of oxygen use. The data limit our cost analysis to direct costs, but because indirect costs of COPD are also substantial, ${ }^{5,15,27}$ the health care costs presented here underestimate the overall burden of COPD. In addition, clinical measures of COPD severity, such as spirometry, were not available in the claims data to validate the cohort classification based on the level of care the patients received. With regard to the analyses by race, differences in health status and treatment patterns, as well as differences in care-seeking behavior, could affect the reported observations. The causal contributions of these factors cannot be discerned from this study. Claims data enabled us to focus on commercial managed care patients, but our findings might not generalize to patients with other types of health care plans, most notably Medicare. Finally, the extrapolation method assumes that the distribution of COPD diagnoses and costs of the population in the research database are similar to the US managed care population. 


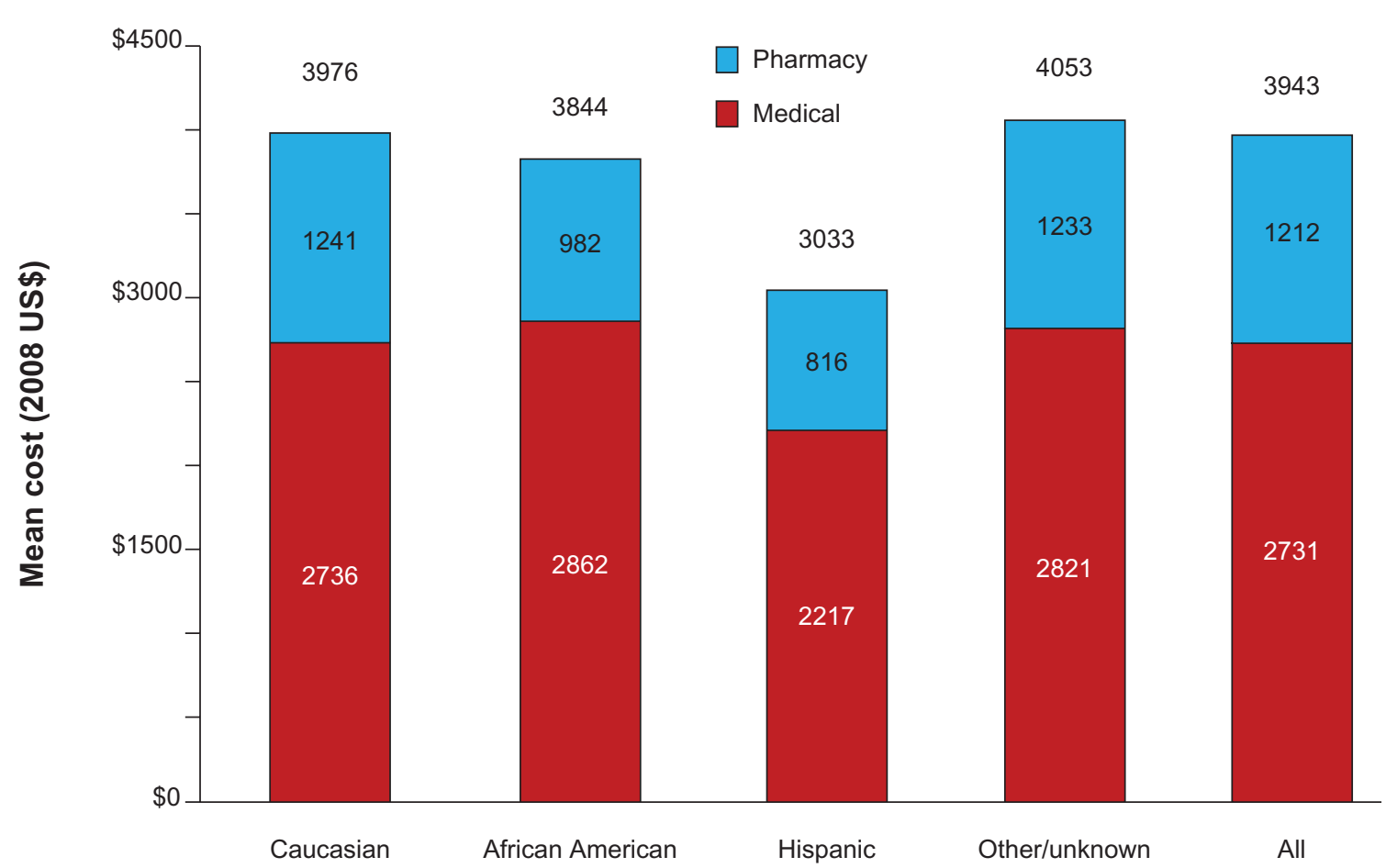

Figure 3 Mean annual patient-level COPD-related health care costs by race. Among-group $P<0.001$ for pharmacy costs, $P=0.58$ I for medical costs, $P=0.135$ for combined medical and pharmacy costs.

Abbreviation: COPD, chronic obstructive pulmonary disease.

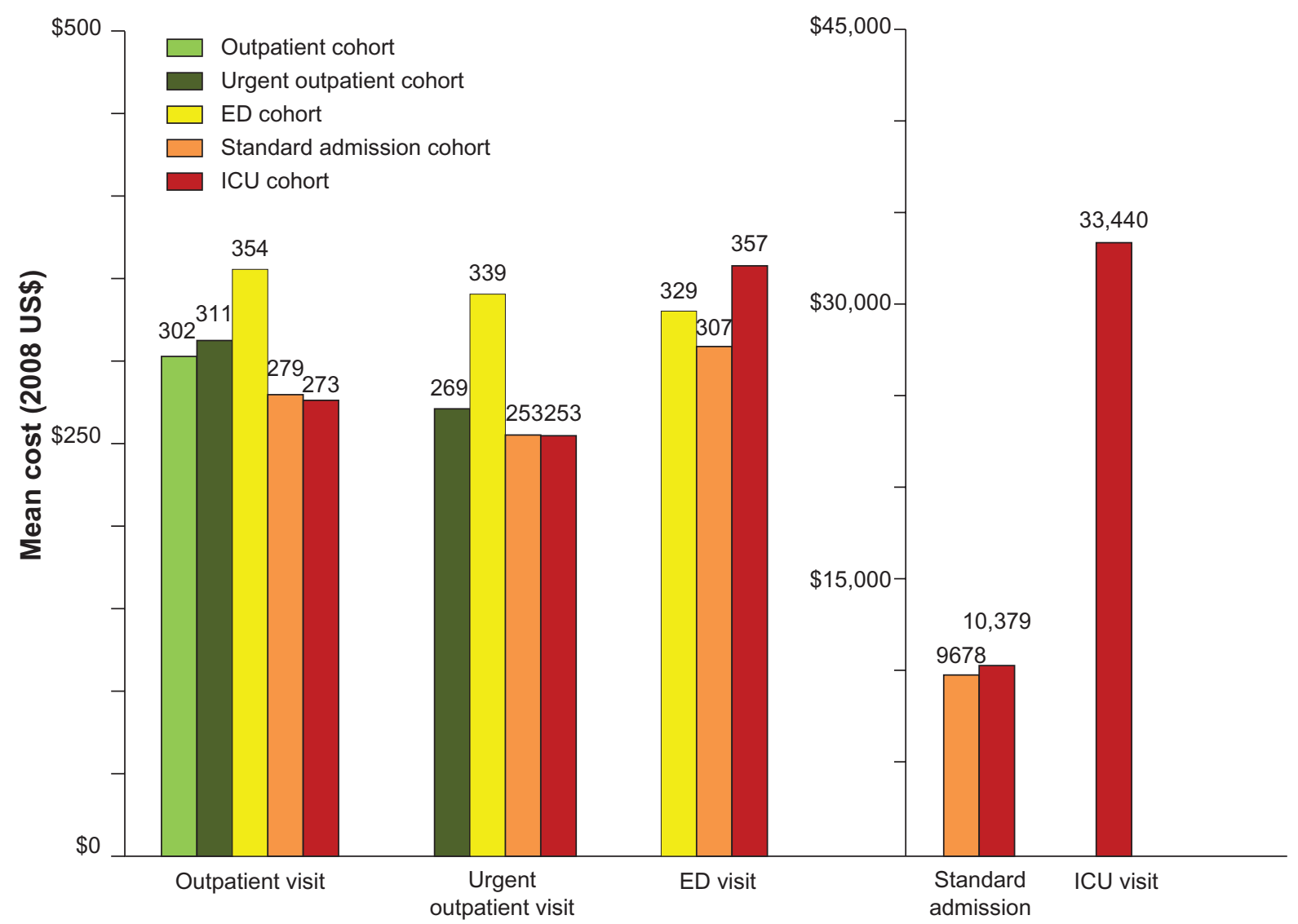

Figure 4 Adjusted episode-level cost of COPD-related visits.

Abbreviations: ED, emergency department; ICU, intensive care unit; COPD, chronic obstructive pulmonary disease. 


\section{Conclusion}

COPD imposes a substantial direct cost burden on patients and commercial payers. Total direct health care costs are driven by medical costs, and COPD-related medical resource use may be considered a proxy for exacerbations. Patients with care indicative of more severe exacerbations had both higher episode- and patient-level costs. These findings suggest that improved symptom management and reduction in exacerbations would reduce the need for escalated care and subsequently reduce overall costs.

\section{Disclosure}

Anand Dalal is an employee of GlaxoSmithKline. All other authors are employees of 13 Innovus, which was contracted by GlaxoSmithKline to conduct the study. Elizabeth J Davis, $\mathrm{PhD}$, i3 Innovus, Eden Prairie, MN, provided medical writing assistance.

\section{References}

1. Global Initiative for Chronic Obstructive Lung Disease (GOLD). Global strategy for the diagnosis, management and prevention of chronic obstructive pulmonary disease. Global Initiative for Chronic Obstructive Lung Disease 2009; Available at: http://www.goldcopd.com/Guidelineitem.asp?11=2\&12=1\&intId=2003. Accessed 2010 Apr 15 .

2. Donaldson GC, Seemungal TA, Patel IS, Lloyd-Owen SJ, Wilkinson TM, Wedzicha JA. Longitudinal changes in the nature, severity and frequency of COPD exacerbations. Eur Respir J. 2003;22(6):931-936.

3. Llor C, Molina J, Naberan K, Cots JM, Ros F, Miravitlles M. Exacerbations worsen the quality of life of chronic obstructive pulmonary disease patients in primary healthcare. Int J Clin Pract. 2008;62(4): 585-592.

4. Anzueto A, Leimer I, Kesten S. Impact of frequency of COPD exacerbations on pulmonary function, health status and clinical outcomes. Int J Chron Obstruct Pulmon Dis. 2009;4:245-251.

5. National Heart Lung and Blood Institute. Morbidity and Mortalitity: Chart Book on Cardiovascular, Lung, and Blood Diseases. National Institutes of Health. 2009; Available at: http://www.nhlbi.nih.gov/resources/docs/ cht-book.htm. Accessed 2010 Apr 15.

6. Mannino DM, Homa DM, Akinbami LJ, Ford ES, Redd SC. Chronic obstructive pulmonary disease surveillance - United States, 1971-2000. MMWR Surveill Summ. 2002;51(6):1-16.

7. Lopez AD, Shibuya K, Rao C, et al. Chronic obstructive pulmonary disease: Current burden and future projections. Eur Respir J. 2006;27(2):397-412.

8. Mathers CD, Loncar D. Projections of global mortality and burden of disease from 2002 to 2030. PLoS Med. 2006;3(11):e442.

9. Celli BR, MacNee W. Standards for the diagnosis and treatment of patients with COPD: A summary of the ATS/ERS position paper. Eur Respir J. 2004;23(6):932-946.

International Journal of COPD

\section{Publish your work in this journal}

The International Journal of COPD is an international, peer-reviewed journal of therapeutics and pharmacology focusing on concise rapid reporting of clinical studies and reviews in COPD. Special focus is given to the pathophysiological processes underlying the disease, intervention programs, patient focused education, and self management protocols.
10. Chronic obstructive pulmonary disease. National clinical guideline on management of chronic obstructive pulmonary disease in adults in primary and secondary care. Thorax. 2004;59 Suppl 1:1-232.

11. Caramori G, Adcock IM, Papi A. Clinical definition of COPD exacerbations and classification of their severity. South Med J. 2009;102(3): 277-282.

12. Stein BD, Charbeneau JT, Lee TA, et al. Hospitalizations for acute exacerbations of chronic obstructive pulmonary disease: How you count matters. COPD. 2010;7(3):164-171.

13. Seemungal T, Stockley R, Calverley P, Hagan G, Wedzicha JA. Investigating new standards for prophylaxis in reduction of exacerbations - the INSPIRE study methodology. COPD. 2007;4(3):177-183.

14. Mittmann N, Kuramoto L, Seung SJ, Haddon JM, Bradley-Kennedy C, Fitzgerald JM. The cost of moderate and severe COPD exacerbations to the Canadian healthcare system. Respir Med. 2008;102(3):413-421.

15. Halpern MT, Stanford RH, Borker R. The burden of COPD in the USA: Results from the Confronting COPD survey. Respir Med. 2003;97 Suppl C:S81-S89.

16. Jansson SA, Andersson F, Borg S, Ericsson A, Jonsson E, Lundback B. Costs of COPD in Sweden according to disease severity. Chest. 2002; 122(6):1994-2002.

17. Andersson F, Borg S, Jansson SA, et al. The costs of exacerbations in chronic obstructive pulmonary disease (COPD). Respir Med. 2002;96(9):700-708.

18. Wouters EF. Economic analysis of the Confronting COPD survey: An overview of results. Respir Med. 2003;97 Suppl C:S3-S14.

19. Agency for Healthcare Research and Quality (AHRQ). Medical Expenditure Panel Survey (MEPS). US Department of Health and Human Services 2006; Available at: http://www.meps.ahrq.gov/mepsweb/. Accessed 2010 Apr 23.

20. Health Insurance Portability and Accountability Act of 1996. Public law 104-191, 104th Congress. US Department of Health and Human Services 1996; Available at: http://www.cms.hhs.gov/HIPAAGenInfo/ Downloads/HIPAALaw.pdf. Accessed 2010 Jan 12.

21. Quan H, Sundararajan V, Halfon P, et al. Coding algorithms for defining comorbidities in ICD-9-CM and ICD-10 administrative data. Med Care. 2005;43(11):1130-1139.

22. US Department of Labor Bureau of Labor Statistics. Consumer Price Index. Chained consumer price index for all urban consumers (C-CPI-U) 1999-2008, Medical Care. Series ID: SUUR0000SAM. Washington, DC: US Department of Labor 2008; Available at: http://data.bls.gov/ cgi-bin/surveymost?su. Accessed 2010 Mar 17.

23. Manning WG. The logged dependent variable, heteroscedasticity, and the retransformation problem. J Health Econ. 1998;17(3):283-295.

24. Stanford RH, Shen Y, McLaughlin T. Cost of chronic obstructive pulmonary disease in the emergency department and hospital: An analysis of administrative data from 218 US hospitals. Treat Respir Med. 2006;5(5):343-349.

25. DalalAA, Shah MS, D'Souza AO, Rane P. Costs of chronic obstructive pulmonary disease exacerbations in the emergency department and inpatient setting. Respir Med. 2010; In press. DOI: 10.1016/j.rmed.2010.09.003.

26. Cecins N, Geelhoed E, Jenkins SC. Reduction in hospitalisation following pulmonary rehabilitation in patients with COPD. Aust Health Rev. 2008;32(3):415-422.

27. Gerdtham UG, Andersson LF, Ericsson A, et al. Factors affecting chronic obstructive pulmonary disease (COPD)-related costs: A multivariate analysis of a Swedish COPD cohort. Eur J Health Econ. 2009; 10(2):217-226.

This journal is indexed on PubMed Central, MedLine and CAS. The manuscript management system is completely online and includes a very quick and fair peer-review system, which is all easy to use. Visit http://www.dovepress.com/testimonials.php to read real quotes from published authors. 